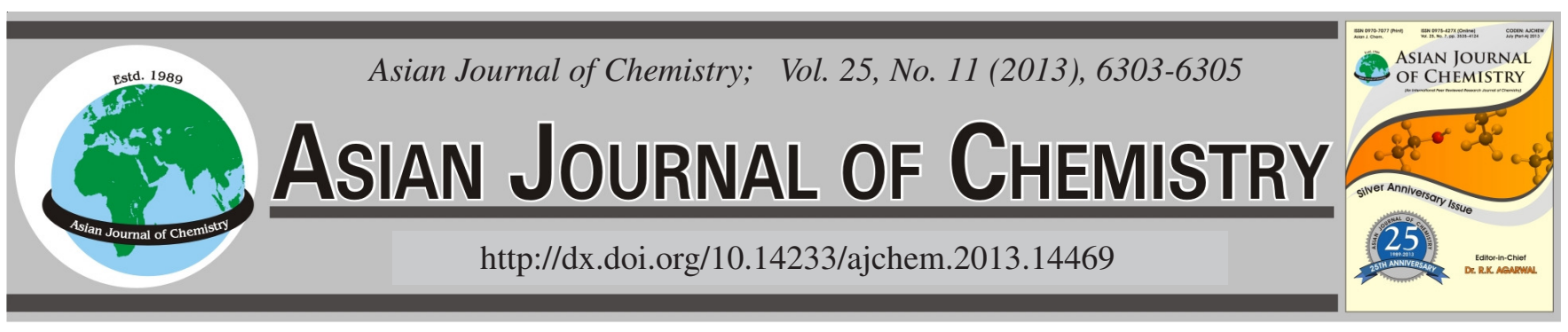

\title{
Iron-Catalyzed Cross-Coupling Reaction of Alkyl Halides with Biphenyl Grignard Reagent
}

\author{
Z.Q. DAI, K.Q. LiU, Z.Y. ZhANG ${ }^{*}$ B.M. WeI and J.T. GuAN
}

School of Chemical and Environmental Engineering, Wuhan Polytechnic University, Wuhan 430023, P.R. China

*Corresponding author: Fax: +86 27 83943956; Tel: +86 13554263937; E-mail: zhiqundai@ 126.com

(Received: 22 August 2012;

Accepted: 8 May 2013)

AJC-13460

\begin{abstract}
In the presence of a catalytic amount of iron salts and $\mathrm{N}, \mathrm{N}, \mathrm{N}^{\prime}, \mathrm{N}^{\prime}$-tetramethylethylene diamine as additive, alkyl bromide reacted with biphenyl magnesium bromide to obtain the cross-coupling product in good yields. The suitable amount of catalyst and the additive are $5 \%$ mol (based on alkyl bromide), 1.3 equiv(based on alkyl bromide), respectively. Under the optimal conditions, the yields of the crosscoupling could reach up to $92.3 \%$.
\end{abstract}

Key Words: Cross-coupling, Grignard reagent.

\section{INTRODUCTION}

The cross-coupling of Grignard reagents with alkyl halides catalyzed by metal is the important method for constructing carbon-carbon bonds in organic synthesis ${ }^{1-3}$. While the coupling reaction of alkyl halides with certain alkyl ${ }^{4-7}$, phenyl or substituted phenyl ${ }^{8-10}$ Grignard reagents is known in the literature, there have been no examples reported of this reaction using biphenyl Grignard reagents. We have recently reported that $\mathrm{CuCl}_{2}{ }^{11}$ and $\mathrm{CuI}^{12}$ catalyzes the cross-coupling reaction of alkyl bromides with biphenylmagnesium bromide in THF for $6 \mathrm{~h}$, the product, alkylbiphenyl, was obtained in high yield; however, shorten the reaction time, unsatisfactory results were obtained. During the course of our study on the cross-coupling reaction of biphenyl Grignard reagents, we have found that $\mathrm{FeCl}_{3}$ show high catalytic reactivity for the cross-coupling reaction of alkyl halides with biphenylmagnesium bromide, the cross-coupling reaction was completed in very short time in high yields in the presence of catalytic amounts of $\mathrm{FeCl}_{3}$ and N,N,N',N'-tetramethylethylene diamine (TMEDA) as an additive (Scheme-I).

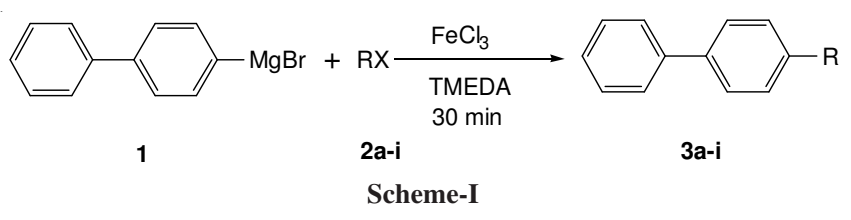

\section{EXPERIMENTAL}

Melting points were determined on a Laboratory Devices Mel-temp apparatus and uncorrected. The IR spectra $(\mathrm{KBr})$ were recorded on a NICOLET 330 FT-IR spectrophotometer; the ${ }^{1} \mathrm{H}$ NMR spectra were measured in a $\mathrm{CDCl}_{3}$ solution with TMS as internal reference on a Varian Mercury VX-400 NMR spectrometer. THF was purified by distillation from sodium prior to use.

General procedure: A mixture of biphenylmagnesium bromide 1 (0.9 $\mathrm{M}$ in THF, $65 \mathrm{mmol})$ and TMEDA $(0.9 \mathrm{M}$ in THF, $65 \mathrm{mmol}$ ) was added to a mixture of alkyl halide $\mathbf{2 a - i}$ (50 mmol) and $\mathrm{FeCl}_{3}(0.1 \mathrm{M}$ in THF, $5 \mathrm{~mol} \%)$ at $-5^{\circ} \mathrm{C}$. The reaction mixture was stirred at $-5{ }^{\circ} \mathrm{C}$ for $0.5 \mathrm{~h}$ and after the completion of the addition of biphenylmagnesium bromide and TMEDA. $1 \mathrm{M} \mathrm{HCl}(50 \mathrm{~mL})$ were added and extracted with toluene $(3 \mathrm{~mL} \times 15 \mathrm{~mL})$. The combined extracts were dried over anhydrous $\mathrm{MgSO}_{4}$. After evaporation of the solvent under the reduced pressure and again the crude products were purified by crystallization from ethanol or distilled under the reduced pressure.

4-Ethylbiphenyl (3a): m.p. $34-35^{\circ} \mathrm{C} ;{ }^{1} \mathrm{H}$ NMR $\left(\mathrm{CDCl}_{3}\right.$, $\delta \mathrm{ppm}): 1.40\left(\mathrm{t}, 3 \mathrm{H},-\mathrm{CH}_{3}\right), 2.52\left(\mathrm{~m}, 2 \mathrm{H},-\mathrm{CH}_{2}-\right), 7.20-7.54$ (m, 9H, ArH); IR (KBr, $\left.v_{\max }, \mathrm{cm}^{-1}\right): 3010,2950,1600,1505$, 1462, 1228, 805, 735; MS (50 eV) m/z (\%): $182\left(\mathrm{M}^{+}, 100\right)$, 167 (75). Anal. calcd. (\%) for $\mathrm{C}_{14} \mathrm{H}_{14}$ : C 92.31, $\mathrm{H}$ 7.69; found (\%) C 92.26, H 7.73.

4-Propylbiphenyl (3b): b.p. $162-165{ }^{\circ} \mathrm{C} / 6 \mathrm{~mm} \mathrm{Hg} ;{ }^{1} \mathrm{H}$ $\mathrm{NMR}\left(\mathrm{CDCl}_{3}, \delta \mathrm{ppm}\right): 1.06\left(\mathrm{t}, 3 \mathrm{H},-\mathrm{CH}_{3}\right), 1.58\left(\mathrm{~m}, 2 \mathrm{H},-\mathrm{CH}_{2-}\right.$ ), 2.55 (t, 2H, - $\mathrm{CH}_{2}-\mathrm{Ar}$ ), 7.20-7.54 (m, 9H, ArH); IR (KBr, $\left.V_{\max }, \mathrm{cm}^{-1}\right): 3050,2895,1605,1508,1456,1280,835,730$; MS (50 eV) m/z (\%): $196\left(\mathrm{M}^{+}, 100\right), 167$ (65). Anal. calcd. (\%) for $\mathrm{C}_{15} \mathrm{H}_{16}$ : C 91.84, H 8. 16; found (\%) C 91.86, H 8.11.

4-Butylbiphenyl (3c): b.p. $140-141^{\circ} \mathrm{C} / 3 \mathrm{~mm} \mathrm{Hg}$ (Lit. ${ }^{12}$ $\left.140{ }^{\circ} \mathrm{C} / 3 \mathrm{mmHg}\right) ;{ }^{1} \mathrm{H}$ NMR $\left(\mathrm{CDCl}_{3}, \delta \mathrm{ppm}\right): 0.91(\mathrm{t}, 3 \mathrm{H}$, $\left.-\mathrm{CH}_{3}\right), 1.34-1.42\left(\mathrm{~m}, 2 \mathrm{H},-\mathrm{CH}_{2^{-}}\right), 1.62-1.67$ (m, $\left.2 \mathrm{H},-\mathrm{CH}_{2^{-}}\right)$, 
2.65 (t, 2H, - $\left.\mathrm{CH}_{2}-\mathrm{Ar}\right), 7.22-7.56$ (m, 9H, ArH); IR (KBr, $\mathrm{v}_{\max }$, $\left.\mathrm{cm}^{-1}\right): 3050,2893,1605,1511,1455,1280,835,745$; MS (50 eV) m/z (\%): $210\left(\mathrm{M}^{+}, 100\right), 167$ (85). Anal. calcd. (\%) for $\mathrm{C}_{16} \mathrm{H}_{18}$ : C 91.37, H 8.63; found (\%) C 91.44, H 8.66.

4-Hexylbiphenyl (3d): m.p. 29-31 ${ }^{\circ} \mathrm{C}$ (Lit. ${ }^{11} 29-32{ }^{\circ} \mathrm{C}$ ); ${ }^{1} \mathrm{H}$ NMR $\left(\mathrm{CDCl}_{3}, \delta \mathrm{ppm}\right): 0.83\left(\mathrm{t}, 3 \mathrm{H},-\mathrm{CH}_{3}\right), 1.22-1.35(\mathrm{~m}$, $\left.6 \mathrm{H},-\left(\mathrm{CH}_{2}\right)_{3}-\right), 1.59$ (t, 2H, $\left.-\mathrm{CH}_{2}-\right), 2.54$ (t, $\left.2 \mathrm{H},-\mathrm{CH}_{2}-\mathrm{Ar}\right), 7.15-$ 7.58 (m, 9H, ArH); IR (KBr, $\left.v_{\max }, \mathrm{cm}^{-1}\right): 3045,2923,1605$, 1501, 1438, 1240.

4-Octylbiphenyl (3e): m.p. $41-42{ }^{\circ} \mathrm{C}$ (Lit. ${ }^{11} 41-42{ }^{\circ} \mathrm{C}$ ); ${ }^{1} \mathrm{H}$ NMR $\left(\mathrm{CDCl}_{3}, \delta \mathrm{ppm}\right): 0.87\left(\mathrm{t}, 3 \mathrm{H},-\mathrm{CH}_{3}\right), 1.27-1.35(\mathrm{~m}$, $\left.10 \mathrm{H},-\left(\mathrm{CH}_{2}\right)_{5^{-}}\right), 1.60\left(\mathrm{t}, 2 \mathrm{H},-\mathrm{CH}_{2^{-}}\right), 2.61\left(\mathrm{t}, 2 \mathrm{H},-\mathrm{CH}_{2}-\mathrm{Ar}\right)$, 7.25-7.60 (m, 9H, ArH); IR (KBr, $\left.v_{\max }, \mathrm{cm}^{-1}\right): 3026,2863,1601$, 1495, 1432, 1283.

4-Nonylbipheny (3f): m.p. $44-46{ }^{\circ} \mathrm{C}$ (Lit. ${ }^{11} 44-45^{\circ} \mathrm{C}$ ); ${ }^{1} \mathrm{H} \mathrm{NMR}\left(\mathrm{CDCl}_{3}, \delta \mathrm{ppm}\right): 0.85\left(\mathrm{t}, 3 \mathrm{H},-\mathrm{CH}_{3}\right), 1.25-1.31(\mathrm{~m}$, $\left.12 \mathrm{H},-\left(\mathrm{CH}_{2}\right)_{6^{-}}\right), 1.57\left(\mathrm{~m}, 2 \mathrm{H},-\mathrm{CH}_{2^{-}}\right), 2.63\left(\mathrm{t}, 2 \mathrm{H},-\mathrm{CH}_{2}-\mathrm{Ar}\right)$, 7.22-7.53 (m, 9H, ArH); IR (KBr, $\left.v_{\max }, \mathrm{cm}^{-1}\right)$ : 3020, 2885, 1600, 1491, 1452, 1280.

4-Decylbiphenyl (3g): m.p. $52-53{ }^{\circ} \mathrm{C}$ (Lit. ${ }^{11} 52-54{ }^{\circ} \mathrm{C}$ ); ${ }^{1} \mathrm{H}$ NMR $\left(\mathrm{CDCl}_{3}, \delta \mathrm{ppm}\right): 0.86\left(\mathrm{t}, 3 \mathrm{H},-\mathrm{CH}_{3}\right), 1.25-1.34(\mathrm{~m}$, $\left.14 \mathrm{H},-\left(\mathrm{CH}_{2}\right)_{7^{-}}\right), 1.63\left(\mathrm{t}, 2 \mathrm{H},-\mathrm{CH}_{2^{-}}\right), 2.65\left(\mathrm{t}, 2 \mathrm{H},-\mathrm{CH}_{2}-\mathrm{Ar}\right)$, 7.25-7.53 (m, 9H, ArH); IR (KBr, $\left.v_{\max }, \mathrm{cm}^{-1}\right)$ : 3045, 2945, 1601, 1495, 1455, 1281.

4-Undecylbiphenyl (3h): m.p. $52-54{ }^{\circ} \mathrm{C}\left(\right.$ Lit. $\left.^{11}{ }^{53-54}{ }^{\circ} \mathrm{C}\right)$; ${ }^{1} \mathrm{H} \mathrm{NMR}\left(\mathrm{CDCl}_{3}, \delta \mathrm{ppm}\right): 0.85\left(\mathrm{t}, 3 \mathrm{H},-\mathrm{CH}_{3}\right), 1.23-1.31(\mathrm{~m}$, $\left.16 \mathrm{H},-\left(\mathrm{CH}_{2}\right)_{8^{-}}\right), 1.52\left(\mathrm{~m}, 2 \mathrm{H},-\mathrm{CH}_{2^{-}}\right), 2.61\left(\mathrm{t}, 2 \mathrm{H},-\mathrm{CH}_{2}-\mathrm{Ar}\right)$, 7.21-7.48 (m, 9H, ArH); IR(KBr, $\left.v_{\max }, \mathrm{cm}^{-1}\right): \delta 3035,2911$, 1590, 1504, 1320, 854, 746.

4-Dodecylbiphenyl (3i): m.p. $61-62^{\circ} \mathrm{C}$ (Lit. $\left.{ }^{11} 61-62{ }^{\circ} \mathrm{C}\right)$; ${ }^{1} \mathrm{H} \mathrm{NMR}\left(\mathrm{CDCl}_{3}, \delta \mathrm{ppm}\right): 0.89\left(\mathrm{t}, 3 \mathrm{H},-\mathrm{CH}_{3}\right), 1.26-1.36(\mathrm{~m}$, $\left.18 \mathrm{H},-\left(\mathrm{CH}_{2}\right)_{9^{-}}\right), 1.62\left(\mathrm{t}, 2 \mathrm{H},-\mathrm{CH}_{2^{-}}\right), 2.64\left(\mathrm{t}, 2 \mathrm{H},-\mathrm{CH}_{2}-\mathrm{Ar}\right)$, 7.25-7.58 (m, 9H, ArH); IR (KBr, $\left.v_{\max }, \mathrm{cm}^{-1}\right): 3040,2931,1609$, $1458,854,746$.

\section{RESULTS AND DISCUSSION}

Initially, we performed the coupling reaction of biphenyl magnesium bromide with $n-\mathrm{C}_{12} \mathrm{H}_{25} \mathrm{Br}$ as a model reaction. Selected results are shown in Table-1. Without any additive, the product was obtained in only $2.1 \%$ yield (Table-1, entry 1). Under similar conditions, tertiary monoamines such as triethylamine and N-methyl morpholine were ineffective (entries 2 and 3) and TMEDA showed high activity for this cross-coupling reaction (entries 4-6). Optimization of the reaction conditions using TMEDA and $\mathrm{FeCl}_{3}$ revealed that use of $5 \mathrm{~mol} \% \mathrm{FeCl}_{3}(0.1 \mathrm{M}$ in THF) and 1.3 equiv of TMEDA ( $0.9 \mathrm{M}$ in THF) based on the halides at $-5^{\circ} \mathrm{C}$ afforded coupling products in highest yield (entry 5).

Further examination of the reaction conditions revealed that slow addition of a mixture of the Grignard reagent and the TMEDA to a solution of the halide and the iron catalyst improves the product yield significantly. The mixture of the Grignard reagent and TMEDA was added at such a rate that the temperature of the react mixture was kept at $-5^{\circ} \mathrm{C}$. The reaction mixture turns dark red and a greatly reduced yield is obtained above $0{ }^{\circ} \mathrm{C}$. The scope of the reaction is illustrated in Table-2.

\begin{tabular}{|c|c|c|c|}
\hline & $\begin{array}{r}\text { CROSS } \\
\text { PHENYLMAGI }\end{array}$ & $\begin{array}{l}\text { TABLE-1 } \\
\text { SUPLING REACTION OF } \\
\text { SIUM BROMIDE WITH n-C }\end{array}$ & $\mathrm{H}_{25} \mathrm{Br}^{\mathrm{a}}$ \\
\hline Entry & $\mathrm{FeCl}_{3}(\mathrm{~mol} \mathrm{\%})^{\mathrm{b}}$ & Additive (equiv.) ${ }^{\mathrm{b}}$ & Yield $(\%)^{\mathrm{c}}$ \\
\hline 1 & 5 & None & 2.1 \\
\hline 2 & 5 & $\mathrm{Et}_{3} \mathrm{~N}(1.3)$ & 8.3 \\
\hline 3 & 5 & N-Methyl morpholine (1.3) & 6.5 \\
\hline 4 & 5 & TMEDA(1.1) & 85.2 \\
\hline 5 & 5 & TMEDA(1.3) & 92.3 \\
\hline 6 & 5 & TMEDA(1.5) & 88.4 \\
\hline 7 & 1 & TMEDA(1.3) & 86.7 \\
\hline 8 & 3 & $\operatorname{TMEDA}(1.3)$ & 90.1 \\
\hline \multicolumn{4}{|c|}{ 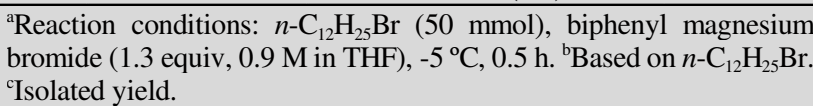 } \\
\hline
\end{tabular}

\begin{tabular}{|c|c|c|c|}
\hline \multicolumn{4}{|c|}{$\begin{array}{c}\text { TABLE-2 } \\
\text { CROSS-COUPLING REACTION OF ALKYL HALIDES } \\
\text { WITH BIPHENYLMAGNESIUM BROMIDE }\end{array}$} \\
\hline Entry & Alkyl halides & Product & Yield $(\%)^{b}$ \\
\hline 1 & $\mathrm{C}_{2} \mathrm{H}_{5}-\mathrm{Br}$ & (3a) & 90.1 \\
\hline 2 & $n-\mathrm{C}_{3} \mathrm{H}_{7}-\mathrm{Br}$ & & 89.3 \\
\hline 3 & $n-\mathrm{C}_{4} \mathrm{H}_{9}-\mathrm{Br}$ & & 89.2 \\
\hline 4 & $n-\mathrm{C}_{6} \mathrm{H}_{13}-\mathrm{Br}$ & $(3 d)$ & 90.4 \\
\hline 5 & $n-\mathrm{C}_{8} \mathrm{H}_{17}-\mathrm{Br}$ & (3e) & 90.6 \\
\hline 6 & $n-\mathrm{C}_{9} \mathrm{H}_{19}-\mathrm{Br}$ & (3f) & 88.8 \\
\hline 7 & $n-\mathrm{C}_{10} \mathrm{H}_{21}-\mathrm{Br}$ & (3g) & 90.1 \\
\hline 8 & $n-\mathrm{C}_{11} \mathrm{H}_{23}-\mathrm{Br}$ & (3h) & 90.5 \\
\hline 9 & $n-\mathrm{C}_{12} \mathrm{H}_{25}-\mathrm{Br}$ & (3i) & 92.3 \\
\hline
\end{tabular}

${ }^{a}$ Reaction conditions: alkyl halides $(50 \mathrm{mmol})$, biphenylmagnesium bromide (1.3 quiv, $0.9 \mathrm{M}$ in THF), TMEDA (1.3 equiv, $0.9 \mathrm{M}$ in $\mathrm{THF}), \mathrm{FeCl}_{3}(0.1 \mathrm{M}$ in THF, $5 \mathrm{~mol} \%),-5^{\circ} \mathrm{C}, 0.5 \mathrm{~h}$. ${ }^{\mathrm{b}}$ Isolated yield.

\section{Conclusion}

For the first time, we have developed the $\mathrm{FeCl}_{3} / \mathrm{TMEDA}$ catalyzed coupling reaction of biphenylmagnesium bromide with alkyl bromide. Good to excellent yields of the coupling products were obtained.

\section{ACKNOWLEDGEMENTS}

The authors are grateful to the National Natural Science Foundation of China (No. 11074054), Foundation of Hubei Educational Commission (No. B20111702), Foundation of Hubei Educational Commission for Distinguished Young Scholars (No. Q20111740) for financial support. 


\section{REFERENCES}

1. V. Bonnet, F. Mongin, F. Trécourt, G. Quéguiner and P. Knochel, Tetrahedron Lett., 42, 5717 (2001).

2. A.C. Frisch and M. Beller, Angew. Chem., 117, 680 (2005).

3. G. Cahiez, C. Chaboche and M. Jezequel, Tetrahedron, 56, 2733 (2000)

4. D. Hauk, S. Lang and A. Murso, Org. Process Res. Devlop., 10, 733 (2006).

5. J. Terao, A. Ikumi, H. Kuniyasu and N. Kambe, J. Am. Chem. Soc., 125, 5646 (2003).

6. J. Terao, H. Todo, S.A. Begum, H. Kuniyasu and N. Kambe, Angew. Chem. Int. Ed., 46, 2086 (2007).
7. J. Terao, H. Watanabe, A. Ikumi, H. Kuniyasu and N. Kambe, J. Am. Chem. Soc., 124, 4222 (2002).

8. C. Gottardo and A. Aguirre, Tetrahedron Lett., 43, 7091 (2002).

9. J. Quintin, X. Franck, R. Hocquemiller and B. Figadère, Tetrahedron Lett., 43, 3547 (2002).

10. J.W. Dankwardt, J. Organomet. Chem., 690, 932 (2005).

11. G. Wang, Z.Y. Zhang, K.C. Zhang, B.-M. Wei and Z.Q. Dai, Chin. J. Org. Chem., 28, 538 (2008)

12. Z.Q. Dai, W.W. Zhang, Z.Y. Zhang and Y.K. Yang, Asian J. Chem., 23, 4087 (2011). 